\title{
ON THE RATIONALIZATION OF THE CIRCLE
}

\author{
CARLES CASACUBERTA
}

(Communicated by Frederick R. Cohen)

\begin{abstract}
We give an example showing that, for a nilpotent group $G$ and a set of primes $P$, the $P$-localization homomorphism $l: G \rightarrow G_{P}$ need not induce an isomorphism in cohomology with arbitrary (twisted) $\mathbf{Z}_{P}$-module coefficients. From this fact we infer that, in the pointed homotopy category of connected $\mathrm{CW}$-complexes, the inclusion of the subcategory of spaces whose higher homotopy groups are $\mathbf{Z}_{P}$-modules and whose fundamental group is uniquely $P^{\prime}$-radicable does not admit a left adjoint.
\end{abstract}

\section{INTRODUCTION}

The purpose of this note is to give the details and discuss the implications of a counterexample which was mentioned in [3]. Let $G$ be a nilpotent group, $P$ a set of primes (different from the set of all primes), and $l: G \rightarrow G_{P}$ the $P$-localization homomorphism [12]. It is well known that, given a nilpotent action of $G_{P}$ on a $\mathbf{Z}_{P}$-module $A$,

$$
G \stackrel{l}{\rightarrow} G_{P} \stackrel{\omega}{\rightarrow} \operatorname{Aut}(A),
$$

the induced homomorphisms in cohomology (with twisted coefficients via $(0.1)$ )

$$
l^{*}: H^{k}\left(G_{P} ; A\right) \rightarrow H^{k}(G ; A)
$$

are isomorphisms for all $k$ (see [12, Theorem I.4.14]). If one removes the assumption that the action $\omega$ be nilpotent, then this needs no longer be true. We supply a counterexample in $\S 1$ : The group $G$ is chosen to be the group of integers (multiplicatively written); then its $P$-localization (i.e., the group $\mathbf{Z}_{P}$ of $P$-local integers, multiplicatively written) acts by multiplication on the rational group algebra $\mathbf{Q}\left[\mathbf{Z}_{P}\right]$, and it turns out that

$$
H^{2}\left(\mathbf{Z}_{P} ; \mathbf{Q}\left[\mathbf{Z}_{P}\right]\right) \neq 0,
$$

while, of course, $H^{2}\left(\mathbf{Z} ; \mathbf{Q}\left[\mathbf{Z}_{P}\right]\right)=0$ because $\mathbf{Z}$ has cohomological dimension one. Thus $(0.2)$ fails to be an isomorphism for $k=2$ in this case.

On the other hand, we know that $(0.2)$ turns out to be an isomorphism, for all $k$, for a certain broad class of not necessarily nilpotent actions (see

Received by the editors November 21, 1991.

1991 Mathematics Subject Classification. Primary 55P60; Secondary $20 \mathrm{C07.}$ 
Theorem 1.1), including all those in which $\operatorname{im} \omega$ is a torsion group of finite exponent (see $[3,16])$.

Our interest in this question arose in the following context: Let $\mathscr{H}$ denote the pointed homotopy category of connected $\mathrm{CW}$-complexes. For a set of primes $P$, let $\mathscr{D}_{P}$ be the full subcategory of $\mathscr{H}$ of spaces $X$ whose higher homotopy groups $\pi_{i}(X), i \geq 2$, are $\mathbf{Z}_{P}$-modules, and whose fundamental group $\pi_{1}(X)$ is uniquely $P^{\prime}$-radicable (i.e., each of its elements has a unique $p$ th root for every prime $p \notin P$; cf. $[12,17])$. In Example 7.3 of [2] the class $\mathscr{D}_{P}$ was erroneously described as the class of targets (or "local objects") associated to a certain idempotent monad in $\mathscr{H}$. We pointed out the difficulty in [3,4], where we described the class of spaces truly arising in that example of [2]. In the present note, we go further by showing that, in fact, the class $\mathscr{D}_{P}$ cannot be the class of local objects associated to any idempotent monad in $\mathscr{H}$.

Our argument is the following. It is well known that the map

$$
S^{1} \rightarrow K\left(\mathbf{Z}_{P}, 1\right)
$$

induced by the embedding of $\mathbf{Z}$ in $\mathbf{Z}_{P}$ is universal among maps from $S^{1}$ to nilpotent spaces in $\mathscr{D}_{P}$ (cf. [12]). However, the observation (0.3) together with a standard obstruction theory argument (see $\S 2)$ show that $(0.4)$ is not universal in $\mathscr{H}$ among maps from $S^{1}$ to arbitrary spaces in $\mathscr{D}_{P}$. Moreover, we show that no map $f: S^{1} \rightarrow X$ with $X$ in $\mathscr{D}_{P}$ can induce a bijection of pointed homotopy classes of maps $f^{*}:[X, Y] \cong\left[S^{1}, Y\right]$ for all spaces $Y$ in $\mathscr{D}_{P}$, so that the inclusion of $\mathscr{D}_{P}$ in $\mathscr{H}$ does not admit a left adjoint.

When this paper was first written, it was based on the observation that $H^{2}(\mathbf{Q} ; \mathbf{Q}[\mathbf{Q}]) \neq 0$, which had been obtained by hand calculation. This fact had implications on the properties of the rationalization map $S^{1} \rightarrow K(\mathbf{Q}, 1)$, as a special case of the results in $\S 2$. This is the origin of the title of the paper. Later, Dicks and Kropholler indicated how to check, by other methods, that $H^{2}(G ; A[G])$ is indeed different from 0 for every noncyclic subgroup $G$ of $\mathbf{Q}$ and every nonzero abelian group $A$. Thus the paper was rewrittenwithout changing the title-so as to apply, more generally, to an arbitrary set of primes $P$.

\section{Algebraic remarks}

We recall the following result from [3]. It is an improvement of an earlier observation of Reynol [16].

Theorem 1.1. Let $G$ be a nilpotent group, $P$ a set of primes, and $l: G \rightarrow G_{P}$ the $P$-localization homomorphism. Let $G_{P}$ act on a $\mathbf{Z}_{P-m o d u l e ~} A$ in such a way that the semidirect product $A \rtimes G_{P}$ is uniquely $P^{\prime}$-radicable. Then the induced homomorphisms $l^{*}: H^{k}\left(G_{P} ; A\right) \rightarrow H^{k}(G ; A)$ are isomorphisms for all $k$.

All nilpotent $\mathbf{Z}_{P}\left[G_{P}\right]$-modules $A$, as well as many others, satisfy the assumption that the semidirect product $A \rtimes G_{P}$ is uniquely $P^{\prime}$-radicable (cf. [3]). Our goal in this section is to supply a counterexample to Theorem 1.1 when this assumption on the action is removed.

Since we are going to deal with group algebras of the form $\mathbf{Q}[G]$, with $G$ a subgroup of $\mathbf{Q}$, it is convenient to use additive notation for the elements of $\mathbf{Q}$ when they occur as coefficients, but multiplicative notation when $\mathbf{Q}$ is viewed 
as an abstract group. Thus we fix a symbol $\xi$ and consider the group algebra Q[Q] of formal sums

$$
\sum_{q \in \mathbf{Q}} a_{q} \xi^{q}, \quad a_{q} \in \mathbf{Q},
$$

where almost all coefficients $a_{q}$ are zero and the multiplication is given by $\xi^{r} \xi^{s}=\xi^{r+s}$. In particular, the cyclic group generated by $\xi$ is identified with the group of integers $\mathbf{Z}$. Of course, $\mathbf{Q}[\mathbf{Q}]$ comes with a canonical action of $\mathbf{Q}$ by multiplication, which restricts to any subgroup of $\mathbf{Q}$ and will be implicit in the coefficients of the cohomology groups in all of what follows.

As we said in the Introduction, the fact that $H^{2}(G ; \mathbf{Q}[G]) \neq 0$ if $G$ is a noncyclic subgroup of $\mathbf{Q}$ is essentially known. It can be proved by combining techniques from $[11,13]$, or also using $[6,7]$. The argument that we give here was suggested by the referee; it is interesting because it is comparatively simple and self-contained.

Proposition 1.2. Let $G$ be any noncyclic subgroup of $\mathbf{Q}$ containing $\mathbf{Z}$. Denote by $\varphi_{n}: \mathbf{Z} \rightarrow \mathbf{Z}$ the nth power map $\varphi_{n}(\xi)=\xi^{n}$ and by

$$
\left(\varphi_{n}\right)^{*}: H^{1}(\mathbf{Z} ; \mathbf{Q}[G]) \rightarrow H^{1}(\mathbf{Z} ; \mathbf{Q}[G])
$$

the homomorphism induced by $\varphi_{n}$. Then:

(a) $\left(\varphi_{n}\right)^{*}$ is not surjective if $n \geq 2$.

(b) Given two integers $n \geq 2, m \geq 1$, not necessarily distinct, the image of $\left(\varphi_{n m}\right)^{*}$ is properly contained in the image of $\left(\varphi_{m}\right)^{*}$.

(c) $H^{2}(G ; \mathbf{Q}[G])$ is uncountable.

Proof. Note that (a) follows from (b) by taking $m=1$. We next prove (b), in a slightly more general form: Assume given a diagram

$$
\mathbf{Z} \stackrel{\varphi_{m}}{\longrightarrow} \mathbf{Z} \stackrel{\varphi_{n}}{\longrightarrow} \mathbf{Z} \stackrel{\tau}{\longrightarrow} \operatorname{Aut}(\mathbf{Q}[G])
$$

where the action $\tau$ is defined by sending the generator $\xi$ to multiplication by a fixed element $\tau=\xi^{q} \in G, q \neq 0$ (the statement of (b) refers to the special case $\tau=\xi$ ). Thus (1.1) gives rise to a commutative diagram

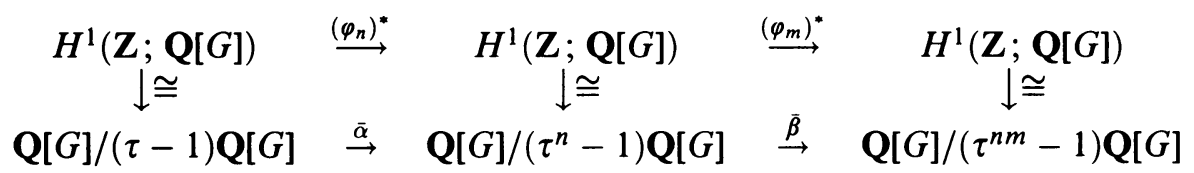

in which the bottom arrows are projections of the endomorphisms of $\mathbf{Q}[G]$ defined, respectively, as multiplication by

$$
\begin{aligned}
& \alpha=1+\tau+\tau^{2}+\cdots+\tau^{n-1}, \\
& \beta=1+\tau^{n}+\tau^{2 n}+\cdots+\tau^{(m-1) n} .
\end{aligned}
$$

Now the class of $\beta$ in $\mathrm{Q}[G] /\left(\tau^{n m}-1\right) \mathrm{Q}[G]$ belongs to the image of $\bar{\beta}$, but not to the image of the composition $\bar{\beta} \bar{\alpha}$. Indeed, suppose that $\beta=\beta \alpha \mu+$ $\left(\tau^{n m}-1\right) \nu$ in $\mathrm{Q}[G]$, for some elements $\mu, \nu$. Then, since $\tau^{n m}-1=$ $\beta \alpha(\tau-1)$, we have $\beta[1-\alpha(\mu+(\tau-1) \nu)]=0$. Since $\mathbf{Q}[G]$ has no zero divisors [15], this tells us that $\alpha$ is a unit in $Q[G]$, which is absurd unless $\tau=1$. This completes the proof of part (b). To prove (c), observe that the 
group $G$ is isomorphic to the direct limit of a certain system

$$
\mathbf{Z} \stackrel{\varphi_{n_{1}}}{\longrightarrow} \mathbf{Z} \stackrel{\varphi_{n_{2}}}{\longrightarrow} \mathbf{Z} \stackrel{\varphi_{n_{3}}}{\longrightarrow} \cdots
$$

in which each $n_{i}$ is an integer bigger than or equal to 2 . The $(k+1)$ th copy of $\mathbf{Z}$ in this system acts on $\mathbf{Q}[G]$ as multiplication by $\xi^{1 / n_{1} \cdots n_{k}}$. Now consider the associated Milnor exact sequence [18, p. 273]

$$
\left.0 \rightarrow \varliminf^{1} H^{1}(\mathbf{Z} ; \mathbf{Q}[G]) \rightarrow H^{2}(G ; \mathbf{Q}[G]) \rightarrow \varliminf^{2} H^{2} \mathbf{Z} ; \mathbf{Q}[G]\right) \rightarrow 0,
$$

and look at the inverse system

$$
H^{1}(\mathbf{Z} ; \mathbf{Q}[G]) \stackrel{\left(\varphi_{n_{1}}\right)^{*}}{\longleftarrow} H^{1}(\mathbf{Z} ; \mathbf{Q}[G]) \stackrel{\left(\varphi_{n_{2}}\right)^{*}}{\longleftarrow} H^{1}(\mathbf{Z} ; \mathbf{Q}[G]) \stackrel{\left(\varphi_{n_{3}}\right)^{*}}{\longleftarrow} \cdots
$$

associated to (1.2). Since $\left(\varphi_{n_{1}}\right)^{*}\left(\varphi_{n_{2}}\right)^{*} \cdots\left(\varphi_{n_{k}}\right)^{*}=\left(\varphi_{n_{1} n_{2} \cdots n_{k}}\right)^{*}$, part (b) ensures that (1.4) is not Mittag-Leffler. Since the groups in (1.4) are countable, Theorem 2 in [14] (see also [10]) tells us that $\varliminf^{1} H^{1}(\mathbf{Z} ; \mathbf{Q}[G])$ is uncountable, which gives the desired result.

Part (a) of Proposition 1.2 is significant in the context of [3], since it shows that the standard map $\varphi_{n}: S^{1} \rightarrow S^{1}$ of degree $n \geq 2$ need not induce an isomorphism in cohomology with arbitrary (twisted) coefficients whose underlying abelian group is a $\mathbf{Q}$-vector space.

In the next section we explain another consequence of Proposition 1.2.

\section{IMPLICATIONS IN HOMOTOPY THEORY}

In what follows, all spaces are assumed to be based connected CW-complexes. We fix a set of primes $P$, different from the set of all primes. The multiplication action of $\mathbf{Z}_{P}$ on $\mathbf{Q}\left[\mathbf{Z}_{P}\right]$ can be realized by an action by basepoint-preserving homeomorphisms of $\mathbf{Z}_{P}$ on an Eilenberg-Mac Lane space $K\left(\mathbf{Q}\left[\mathbf{Z}_{P}\right], 2\right)$. Consider the space

$$
L=E\left(\mathbf{Z}_{P}\right) \times \mathbf{Z}_{P} K\left(\mathbf{Q}\left[\mathbf{Z}_{P}\right], 2\right)
$$

obtained by dividing out the diagonal action of $\mathbf{Z}_{P}$ on $E\left(\mathbf{Z}_{P}\right) \times K\left(\mathbf{Q}\left[\mathbf{Z}_{P}\right], 2\right)$, where $E\left(\mathbf{Z}_{P}\right)$ is the universal cover of a $K\left(\mathbf{Z}_{P}, 1\right)$. This space $L$ has fundamental group isomorphic to $Z_{P}$ and a single nonvanishing higher homotopy group, namely $\pi_{2}$, which is isomorphic to $\mathbf{Q}\left[\mathbf{Z}_{P}\right]$. Thus it belongs to the class $\mathscr{D}_{P}$ of spaces whose higher homotopy groups are $\mathbf{Z}_{P}$-modules and whose fundamental group is uniquely $P^{\prime}$-radicable.

Recall from [5,9] that, given a space $X$ and a group homomorphism $\varphi: \pi_{1}(X) \rightarrow \mathbf{Z}_{P}$, there is a one-to-one correspondence between the second cohomology group $H^{2}\left(X ; \mathbf{Q}\left[\mathbf{Z}_{P}\right]\right)$ with twisted coefficients via $\varphi$ and the set of pointed homotopy classes of maps $f: X \rightarrow L$ inducing $\varphi$ on the fundamental group.

Now suppose that the map $l: K(\mathbf{Z}, 1) \rightarrow K\left(\mathbf{Z}_{P}, 1\right)$ is universal (initial) in $\mathscr{H}$ among maps from $K(Z, 1)$ to spaces in $\mathscr{D}_{P}$. Then, in particular, we have a bijection of pointed homotopy classes of maps

$$
l^{*}:\left[K\left(\mathbf{Z}_{P}, 1\right), L\right] \cong[K(\mathbf{Z}, 1), L],
$$


which restricts to a bijection of classes of maps inducing, respectively, the identity of $\mathbf{Z}_{P}$ and the inclusion $\mathbf{Z} \rightarrow \mathbf{Z}_{P}$ on the fundamental group. This provides a cohomology isomorphism

$$
l^{*}: H^{2}\left(\mathbf{Z}_{P} ; \mathbf{Q}\left[\mathbf{Z}_{P}\right]\right) \cong H^{2}\left(\mathbf{Z} ; \mathbf{Q}\left[\mathbf{Z}_{P}\right]\right)
$$

contradicting Proposition 1.2 and hence proving that $l$ fails to be universal in $\mathscr{H}$. Moreover, we next show that this difficulty cannot be solved by replacing $K\left(\mathbf{Z}_{P}, 1\right)$ by any other space.

Proposition 2.1. The inclusion of $\mathscr{D}_{P}$ in $\mathscr{H}$ does not admit a left adjoint.

Proof. Assume that some map $f: S^{1} \rightarrow X$ is universal in $\mathscr{H}$ among maps from $S^{1}$ to spaces in $\mathscr{D}_{P}$. Then for every uniquely $P^{\prime}$-radicable group $G$ we have a bijection

$$
f^{*}:[X, K(G, 1)] \cong\left[S^{1}, K(G, 1)\right],
$$

which is equivalent to

$$
f^{*}: \operatorname{Hom}\left(\pi_{1}(X), G\right) \cong \operatorname{Hom}(\mathbf{Z}, G),
$$

and this tells us that $f_{*}: \mathbf{Z} \rightarrow \pi_{1}(X)$ is a $P$-equivalence of groups. Since $X$ is in $\mathscr{D}_{P}$, it follows that $\pi_{1}(X) \cong \mathbf{Z}_{P}$. Note that we are using here the easily checked fact that the inclusion $\mathbf{Z} \rightarrow \mathbf{Z}_{P}$ is universal among homomorphisms from $\mathbf{Z}$ to uniquely $P^{\prime}$-radicable groups, not only in the category of nilpotent groups [12] but also in the category of all groups (contrary to what happens in the homotopy-theoretical analogue under discussion!).

Now let $\pi_{1}(X)$ act by multiplication on $\mathrm{Q}\left[\mathbf{Z}_{P}\right]$ and obtain, by the same argument used in (2.2) and (2.3), a bijection $f^{*}:[X, L] \cong\left[S^{1}, L\right]$ restricting to an isomorphism

$$
f^{*}: H^{2}\left(X ; \mathbf{Q}\left[\mathbf{Z}_{P}\right]\right) \cong H^{2}\left(S^{1} ; \mathbf{Q}\left[\mathbf{Z}_{P}\right]\right) .
$$

But for every space $X$ the Serre spectral sequence associated to the universal covering $\widetilde{X} \rightarrow X \rightarrow K\left(\pi_{1}(X), 1\right)$ tells us that $H^{2}\left(\pi_{1}(X) ; A\right)$ embeds as a subgroup in $H^{2}(X ; A)$ for every coefficient module $A$. Thus, by Proposition 1.2, $H^{2}\left(X ; \mathbf{Q}\left[\mathbf{Z}_{P}\right]\right) \neq 0$, which contradicts $(2.4)$.

In [3] we study certain subcategories of $\mathscr{D}_{P}$ whose inclusion in $\mathscr{l}$ does admit a left adjoint. The main examples are the class of $H_{*}\left(; \mathbf{Z}_{P}\right)$-local spaces in the sense of Bousfield [1], and the class of spaces $X$ for which the $p$ th power map $\sigma \mapsto \sigma^{p}$ on the loop space $\Omega X$ is a self-homotopy equivalence for all primes $p \notin P$. It is interesting that these two classes of spaces seem to be respectively minimal and maximal within $\mathscr{D}_{P}$ with the property that a left adjoint of their inclusion into $\mathscr{H}$ exists and sends $S^{1}$ to a $K\left(\mathbf{Z}_{P}, 1\right)$. Note that the space $L$ that we used above, as well as many others, must have been left out of the latter two classes of spaces.

We finally observe that, in the context of the recent work of Dror Farjoun [8], Proposition 2.1 implies that there is no map $f: A \rightarrow B$ in $\mathscr{H}$ such that the class of $f$-local spaces (i.e., spaces $X$ such that $f^{*}: \operatorname{map}_{*}(B, X) \simeq \operatorname{map}_{*}(A, X)$ ) coincides with $\mathscr{D}_{P}$. 


\section{ACKNOWLEDGMENTS}

This note is an appendix to joint work of the author with Georg Peschke and Markus Pfenniger. It arose from discussions with Craig Squier, and was completed while the author was visiting the SFB 170 in Göttingen.

\section{REFERENCES}

1. A. K. Bousfield, The localization of spaces with respect to homology, Topology 14 (1975), 133-150.

2. $\longrightarrow$, Constructions of factorization systems in categories, J. Pure Appl. Algebra 9 (1977), 207-220.

3. C. Casacuberta and G. Peschke, Localizing with respect to self maps of the circle, Trans. Amer. Math. Soc. (to appear).

4. C. Casacuberta, G. Peschke, and M. Pfenniger, Sur la localisation dans les catégories avec une application à la théorie de l'homotopie, C. R. Acad. Sci. Paris Sér. I Math. 310 (1990), 207-210.

5. A. Deleanu and P. Hilton, On Postnikov-true families of complexes and the Adams completion, Fund. Math. 106 (1980), 53-65.

6. W. Dicks, Groups, trees and projective modules, Lecture Notes in Math., vol. 790, SpringerVerlag, Berlin and New York, 1980.

7. W. Dicks and M. J. Dunwoody, Groups acting on graphs, Cambridge Stud. Adv. Math., vol. 17, Cambridge Univ. Press, Cambridge and New York, 1989.

8. E. Dror Farjoun, Homotopy localization and $v_{1}$-periodic spaces, Algebraic Topology; Homotopy and Group Cohomology, Lecture Notes in Math., vol. 1509, Springer-Verlag, Berlin and New York, 1992, pp. 104-113.

9. S. Gitler, Cohomology operations with local coefficients, Amer. J. Math. 85 (1963), 156-188.

10. B. Gray, Spaces of the same n-type for all $n$, Topology 5 (1966), 241-243.

11. K. W. Gruenberg, Cohomological topics in group theory, Lecture Notes in Math., vol. 143, Springer-Verlag, Berlin and New York, 1970.

12. P. Hilton, G. Mislin, and J. Roitberg, Localization of nilpotent groups and spaces, NorthHolland Math. Stud., vol. 15, North-Holland, Amsterdam, 1975.

13. D. F. Holt, The cohomological dimensions of locally finite groups, J. London Math. Soc. (2) 24 (1981), 129-134.

14. C. A. McGibbon and J. M. Møller, On spaces with the same $n$-type for all $n$, Topology 31 (1992), 177-201.

15. D. Passman, The algebraic structure of group rings, Wiley, New York, 1977.

16. A. Reynol, P-localization of some classes of groups, Ph.D. thesis, Universidade de São Paulo, Brazil, 1987.

17. P. Ribenboim, Torsion et localisation de groupes arbitraires, Séminaire d'algèbre Paul Dubreil, Lecture Notes in Math., vol. 740, Springer-Verlag, Berlin and New York, 1979, pp. 444-456.

18. G. W. Whitehead, Elements of homotopy theory, Graduate Texts in Math., vol. 61, SpringerVerlag, New York, 1978.

Departament d’Àlgebra i Geometria, Facultat de Matemàtiques, Universitat de Barcelona, Gran Via de les Corts Catalanes 585, E-08007 Barcelona, Spain

E-mail address: icrm1@cc.uab.es 